\title{
Automated Semi-Quantitative Analysis of Breast MRI: Potential Imaging Biomarker for the Prediction of Tissue Response to Neoadjuvant Chemotherapy
}

\author{
Matthias Dietzel $^{\mathrm{a}}$ Clemens Kaiser $^{\mathrm{b}} \quad$ Katja Pinker ${ }^{\mathrm{c}, \mathrm{d}} \quad$ Evelyn Wenkel $^{\mathrm{a}}$ Matthias Hammon $^{\mathrm{a}}$ \\ Michael Uder ${ }^{a}$ Barbara Bennani Baiti ${ }^{d}$ Paola Clauser $^{d}$ Rüdiger Schulz-Wendtland ${ }^{\mathrm{a}}$ \\ Pascal Baltzer ${ }^{d}$ \\ a Department of Radiology, University Hospital Erlangen-Nürnberg, Erlangen, Germany; \\ ${ }^{b}$ Institute of Clinical Radiology and Nuclear Medicine, University Medical Center Mannheim, Mannheim, Germany; \\ ${ }^{\mathrm{c}}$ Department of Radiology, Molecular Imaging and Therapy Service, Memorial Sloan Kettering Cancer Center, New York, NY, USA; \\ ${ }^{\mathrm{d}}$ Medical University of Vienna, Department of Biomedical Imaging and Image-Guided Therapy, Division of Molecular and \\ Gender Imaging, Vienna, Austria
}

\section{Keywords}

Breast MRI · Primary systemic chemotherapy .

Therapy response $\cdot$ Imaging biomarkers

\section{Summary}

Background: We aimed to investigate an automated semi-quantitative software as an imaging biomarker for the prediction of tissue response (TR) after completion of neoadjuvant chemotherapy (NAC). Methods: Breast magnetic resonance imaging (MRI) (1.5T, protocol according to international recommendations) of 67 patients with biopsy-proven invasive breast cancer were examined before and after NAC. After completion of NAC, histopathologic assessments of TR were classified according to the Chevallier grading system (CG1/4: full/non-responder; CG2/C3: partial responder). A commercially available fully automatic software (CADstream) extracted MRI parameters of tumor extension (tumor diameter/volume: TD/TV). Pre- versus post-NAC values were compared ( $\triangle T V$ and $\triangle T D$ ). Additionally, the software performed volumetric analyses of vascularization (VAV) after NAC. Accuracy of MRI parameters to predict TR were identified (cross-tabs, ROC, AUC, Kruskal-Wallis). Results: There were 37 (34.3\%) CG1, 7 (6.5\%) CG2, 53 (49.1\%) CG3, and $11(10.2 \%)$ CG4 lesions. The software reached area under the curve levels of $79.5 \%$ (CG1/complete response: $\triangle T D$ ), $68.6 \%$ (CG2, CG3/partial response: VAV), and $88.8 \%$ to predict TR (CG4/non-response: $\triangle T V$ ). Conclusion: Semiquantitative automated analysis of breast MRI data enabled the prediction of tissue response to NAC.

(C) 2017 S. Karger GmbH, Freiburg

\section{Introduction}

As major studies have demonstrated equal efficacy of adjuvant and neoadjuvant cytotoxic regimens, neoadjuvant chemotherapy (NAC) is an important treatment option for invasive breast cancer $[1,2]$. There are several advantages of NAC: In patient responding to NAC, the rate of breast-conserving therapy can be increased. Therefore, it is no surprise that S3 guidelines recommend NAC particularly in the setting of advanced or even primarily inoperable cancers [2]. Among further advantages of NAC, the potential to directly evaluate treatment efficacy is probably most important. Hereby, the response rate can be investigated with histological samples, which is impossible in the adjuvant situation. As NAC enables in vivo sensitivity tests to study tissue response (TR), we can individually adopt cytotoxic regimens either by changing the frequency, duration, and dosage of the agent or by switching to another NAC regimen. Non-response to NAC is one of the strongest surrogate parameters of patient outcome and survival $[1,2]$.

In the assessment of TR to NAC, medical imaging plays a central role [3]. Size measurement before, during, and after NAC is one of the most frequently applied methods to assess the response to cytotoxic treatments in daily practice. In this context, mammography, ultrasound, and breast $\mathrm{m}$ agnetic resonance imaging (bMRI) are the standard methods. Ultrasound is easy to assess, cheap, rather fast, and offers high spatial resolution. Therefore, ultrasound is the workhorse in the assessment of TR to NAC in many institutions.

Unlike mammography, bMRI is a 3-dimensional technique without the need of breast compression or exposure to ionizing radiation. Different from ultrasound, bMRI acquisition itself is less user-dependent, and images are easy to document. Previous bMRI

\section{KARGER}

(C) 2017 S. Karger GmbH, Freiburg
Prof. Dr. med. Pascal Baltzer Department of Biomedical Imaging and Image-Guided Therapy Medical University of Vienna

Währinger Gürtel 18-20, 1090 Wien, Austria pascal.baltzer@meduniwien.ac.at 
investigations assessed TR to NAC mainly based on changes in tumor diameter [3]. Marinovich et al. [3] performed a meta-analysis regarding the accuracy of bMRI versus ultrasound and mammography in comparison to the gold standard (mean difference (MD) between bMRI and pathology). Based on a large body of evidence, they concluded that bMRI (MD $0.0 \mathrm{~mm}$ ) is generally superior to ultrasound as well as mammography and should be considered the most appropriate test in the assessment of TR to NAC.

Besides basic tumor size measurement, bMRI provides functional data about tissue vascularization $[4,5]$. As shown in previous research, such data reflect the tumor biology, are connected to patient outcomes [6-8], and could be used as surrogate parameters of TR (for instance [9]). Yet, the major drawback of bMRI is its complex interpretation $[10,11]$. This is particularly challenging in the context of dynamic examinations (e.g. follow-up examination during NAC) and multicentric oncological trials. Accordingly, bMRI is still not routinely used in the assessment of TR to NAC [2].

A standardized method providing both automated measurements and standardized documentation of serial bMRI data that could be used as surrogate parameters of TR would be highly desirable. Therefore, we investigated the accuracy of an automated semi-quantitative software as an imaging biomarker for the prediction of TR after completion of NAC.

\section{Patients and Methods}

\section{Study Design}

For this retrospective, cross-sectional, single-center study, performed at the university hospital of Jena, informed consent was waived by the local institutional ethical committee. Data were collected at an academic tertiary care institution run by interdisciplinary breast cancer specialists (gynecology, oncology, pathology, radiation oncology, and radiology), and therapeutic decisions such as indication for NAC were established by multidisciplinary consensus. Treatment and follow-up were performed following national guidelines [2]. Accordingly, NAC was indicated in the case of locally advanced invasive and inflammatory cancers in order to reduce local tumor burden and hereby increase the rate of breast-conserving surgeries. NAC schemes contained 1 anthracycline and 1 taxane agent plus trastuzumab in the case of positive HER2 testing. NAC schemes were administered in 6-8 cycles over 18-24 weeks.

Primary indications for bMRI were BI-RADS IV or V findings upon mammography and/or breast ultrasound. Out of this patient pool, all consecutive individuals over a period of 24 months with biopsy-proven primary invasive breast cancer (biopsies were performed after bMRI) receiving a complete scheme of NAC according to the above criteria were selected for the present analysis.

\section{Standard of Reference}

Histopathology was performed by 2 board-certified breast pathologists in consensus. The diagnosis of 'breast cancer' was based on specimens from image-guided biopsy before the initiation of NAC. As all lesions were visible in breast ultrasound, 14-gauge core biopsies under sonographic guidance were performed for tissue sampling. After completion of NAC, specimens taken from mastectomy or breast-conserving therapy were used to define the response to treatment. The TR of every single tumor lesion was graded using the Chevallier classification $[12,13]$ (As this study focuses on the direct correlation of bMRI pattern with TR, the axilla was not considered):

Full responder: absence of residual tissue (Chevallier grade 1: CG1).

Partial responder: absence of vital tumor but remaining noninvasive single cancer cells (CG2).

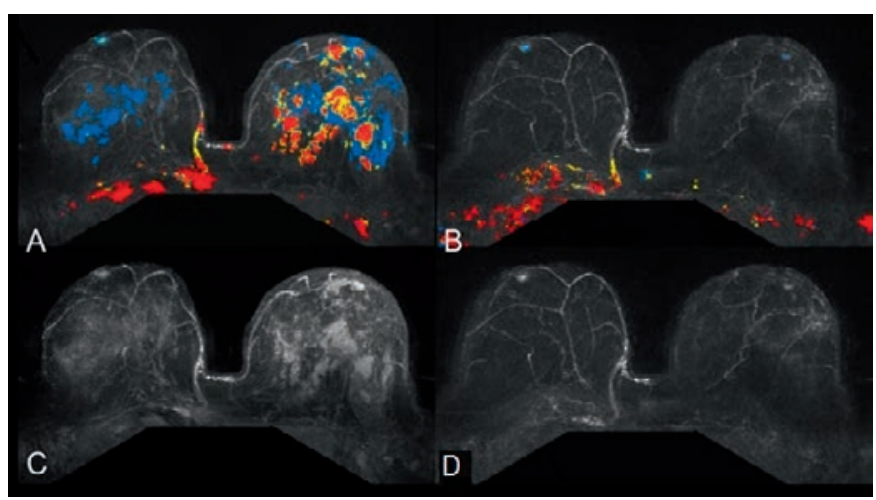

Fig. 1. Clinical example of $\mathrm{CAD}$ (computer-aided diagnosis) analysis in a 45-year-old woman before (A, C) and after neoadjuvant chemotherapy (NAC) (B, D). Histology revealed a multicentric HER2-enriched invasive ductal cancer grade 3 of the left breast. A, B Breast magnetic resonance imaging (bMRI) with color-coded tumor volume. Before NAC (A), the dominating red voxels represent fast wash in with wash out, and yellow represents those with fast wash in and plateau; blue codes voxels with slow wash in and persistent increase. This gives the picture of a heterogeneous tumor with multiple spots of highly pathological vasculature. After NAC (B), bMRI demonstrated significant remission of the tumor foci. The previously distinct bilateral background parenchymal enhancement has resolved. There is only faint residual tumor enhancement mostly below the enhancement threshold (no color coding). 1 residual spot with still increased vascularity in ventral position is marked in blue color by the CAD. Findings were consistent with Chevallier grade 2 which was verified by histopathology. C, D Maximum intensity projection images without color-coded overlay before (C) and after (D) NAC illustrate morphologic findings.

Partial responder: vital tumor present demonstrating changes related to NAC (CG3).

Non-responder: vital tumor present without evidence of changes related to NAC (CG4).

\section{MRI}

Patients were examined before and after the completion of NAC. bMRI Protocols were standardized with exactly the same parameters before and after NAC by one single 1.5 Tesla MRI unit using a vendor-supplied bilateral 4-channel breast coil (Magnetom Symphony, Siemens Healthineers, Erlangen, Germany) [14]. The bMRI protocol according to [14] consisted of one non-fatsaturated morphologic T2-weighted sequence and repetitive radiofrequency spoiled dynamic T1-weighted gradient echo sequences (Fast Low Angle SHot, FLASH 2D) acquired at 1-min intervals over $8 \mathrm{~min}$. Only the dynamic sequences were used for this investigation (see [15] for full diagnostic protocol). After 1 baseline scan, gadopentetate dimeglumine (Magnevist, Bayer/Schering HealthCare, Leverkusen, Germany) was administered into an antecubital vein by a power injector at a dosage of $0.1 \mathrm{mmol} / \mathrm{kg}$ body weight with a flow rate of $3 \mathrm{ml} / \mathrm{s}$, followed by a $20 \mathrm{ml}$ saline flush (Spectris, Medrad, Pittsburgh, PA, USA). Post-contrast scanning started $30 \mathrm{~s}$ after contrast injection using the same adjustment settings. Acquisition parameters were $110 \mathrm{~ms}$ (repetition time), $5 \mathrm{~ms}$ (echo time), $80^{\circ}$ (flip angle), $1.1 \times 0.9 \times 3 \mathrm{~mm}$ (spatial resolution), and $350 \mathrm{~mm}$ (field of view).

\section{MRI Analysis}

Imaging data were processed by dedicated, commercially available, FDAapproved software (CADstream, Confirma, Kirkland, WA, USA). The software automatically segmented the lesions and provided 3 enhancement parameters on a voxel-by-voxel basis. The initial enhancement threshold was set to 40 . Hereby, the initial enhancement defines the signal intensity of the first minute post-contrast normalized (\%) to the baseline signal before gadolinium. 2 experienced bMRI radiologists ( $>500$ examinations/year) blinded to the standard of 
reference supervised the automated software analysis. Both were trained by the vendor's application specialist in handling the software. Figure 1 highlights a typical example of CAD (computer-aided diagnosis) analysis. Here, 3 enhancement parameters were automatically extracted by the software:

Tumor volume (TV) integrated all voxels passing the enhancement threshold. In order to reduce the influence of partial volume effects, a voxel was defined as a cluster of 3 by 3 pixels. Based on the spatial resolution $(1.1 \times 0.9 \times 3 \mathrm{~mm}$, see above), the TV was calculated in $\mathrm{cm}^{3}$.

For tumor diameter (TD), the largest diameter of the TV was identified by the algorithm. Based on the spatial resolution, it was documented in $\mathrm{mm}$.

Volumetric analysis of tumor vascularization (VAV) investigated enhancement characteristics of the tumor after completion of NAC. Typical patterns of the early and delayed phase were analyzed as described by Morris et al. [10]. First, the software automatically identified the most suspicious tumor compartment. This was defined as the tumor voxel showing the highest 'wash in' to 'wash out' ratio within the voxels of interest (VOI). Second, the vascularization of this tumor compartment was categorized as 'slow', 'medium', or 'strong wash in' $(<40 \%, 40-100 \%,>100 \%)$ to describe the initial enhancement, and 'wash out', 'plateau' or 'persistent' to describe the delayed enhancement $(<10 \%,+/-10 \%$, $>10 \%$,) (percentages normalized to the first scan after contrast application). As stated in table 1 , this gave a total of 7 categories labeled $\mathrm{VAV}_{0}$ to $\mathrm{VAV}_{6}$.

\section{Statistical Analysis}

To address the changes related to NAC, $\Delta \mathrm{TV}$ and $\Delta \mathrm{TD}$ were calculated in $\%$ as follows:

Table 1. Summary of computer-aided diagnosis (CAD) parameters used for breast magnetic resonance imaging (bMRI) analysis

\begin{tabular}{ll}
\hline Parameter & Brief description \\
\hline $\begin{array}{l}\text { Tumor volume (TV), } \\
\mathrm{cm}^{3}\end{array}$ & $\begin{array}{l}\text { sum of all tumor voxels showing wash in }>40 \% \\
\text { within the VOI }\end{array}$ \\
\hline $\begin{array}{l}\text { Tumor diameter (TD), } \\
\text { mm }\end{array}$ & maximum diameter of the TV \\
\hline
\end{tabular}

VAV category

\begin{tabular}{llll}
\cline { 2 - 3 } 0 & wash in $<40 \%$ & \\
\cline { 2 - 4 } 1 & wash in between 40 & plus & $\begin{array}{l}\text { persistent } \\
\text { plateau } \\
2\end{array}$ \\
3 & and $100 \%$ & & wash out \\
\hline 4 & & plus & $\begin{array}{l}\text { persistent } \\
\text { plateau } \\
5\end{array}$ \\
6 & wash in $>100 \%$ & & wash out
\end{tabular}

aVAV investigates the voxel within the tumor showing the highest 'wash in' to 'wash out' ratio. Based on the enhancement characteristics of the early (wash in) and late phase of this voxel (persistent, plateau, wash out), VAV was classified into a total of 7 categories $\left(\mathrm{VAV}_{0-6}\right)$.

$\mathrm{VOI}=$ voxels of interest; $\mathrm{VAV}=$ volumetric analysis of tumor vascularization after completion of neoadjuvant chemotherapy.
Non-parametric rank tests (Kruskal-Wallis test; appropriate post-hoc analysis), rank correlation (Spearman's rho), and standard descriptive statistics were performed to explore the association of VAV, $\triangle \mathrm{TV}$, and $\triangle \mathrm{TD}$ with TR.

The association of quantitative parameters $(\Delta \mathrm{TV}, \Delta \mathrm{TD})$ with TR was investigated by ROC (receiver operating characteristics) with subsequent AUC (area under the curve) analysis. Based on appropriate cut-off criteria, corresponding values for sensitivity, specificity, and negative/positive likelihood ratios (L-, L+) were calculated.

The association of the ordinal parameter VAV with TR was explored with logistic regression. Corresponding predicted values were saved and analyzed by AUC analysis just like $\Delta \mathrm{TV}, \Delta \mathrm{TD}$.

For all statistical tests, the null hypothesis (H0) was defined as 'no association between NAC and the given MR parameter'. The analysis was done on a lesion-based level. Hypotheses were tested in a 2-sided manner; a was defined as $5 \%$.

\section{Results}

\section{Patients and Standard of Reference}

In total, 108 histologically verified malignant lesions in $67 \mathrm{pa}-$ tients were included. The mean patient age was $51.7+/-10.3$ years. The mean lesion size was $26.8+/-19.3 \mathrm{~mm}$. There were $92(85.2 \%)$ invasive ductal, 10 (9.3\%) invasive lobular, 5 (4.6\%) inflammatory, and $1(0.9 \%)$ invasive medullary cancers. Tumor grading was G1 in $9(8.3 \%)$, G2 in 41 (38\%), and G3 in 58 (53.7\%) cases. After completion of NAC, Chevallier grading was ' 1 ' in 37 (34.3\%), ' 2 ' in 7 (6.5\%), ' 3 ' in $53(49.1 \%)$, and ' 4 ' in $11(10.2 \%)$ patients. Table 2 gives a cross tabulation of histopathological subgroups (tumor type, grading) and CG.

\section{$\Delta T D, \Delta T V$}

Median values of $\Delta \mathrm{TD}$ were $100 \%$ for CG1, 96\% for CG2, $81 \%$ for CG3, and 36\% for CG4. In the 3 categories, CG1 to CG3 median values of $\Delta \mathrm{TV}$ were $100 \% . \Delta \mathrm{TV}$ of non-responders (CG4) was 75\%. Differentiation of CG2 versus CG3 and CG1 versus CG2 was not possible using either $\Delta \mathrm{TD}$ or $\Delta \mathrm{TV}$ ( $\mathrm{p}=$ not significant (n.s.)). All other CG could be clearly differentiated by both parameters $(\mathrm{p}<0.05)$. For both $\Delta \mathrm{TD}($ rho $=-0.60)$ and $\Delta \mathrm{TV}($ rho $=-0.59)$, there was an inverse correlation with CG (both $\mathrm{p}<0.001$ ).

\section{VAV}

Typically, complete responders (CG1) showed $\mathrm{VAV}_{0}$ (91.9\%, $\mathrm{n}=34 ; \mathrm{p}<0.001)$. The 3 remaining CG1 patients were $\operatorname{VAV}_{1}(\mathrm{n}=2)$ and $\mathrm{VAV}_{5} . \mathrm{VAV}_{6}$ was most frequent $(54.5 \%, \mathrm{n}=6)$ in nonresponders. The 5 remaining CG4 cases were $\mathrm{VAV}_{0}, \mathrm{VAV}_{3}$ (both $\mathrm{n}=1,9.1 \%)$, and $\operatorname{VAV}_{4}(27.3 \%, \mathrm{n}=3)$.

Table 2. Cross tabulation of histopathological subgroups (tumor type, grading) versus Chevallier grade

\begin{tabular}{|c|c|c|c|c|c|c|c|c|}
\hline \multirow{2}{*}{$\begin{array}{l}\text { Chevallier } \\
\text { grade }\end{array}$} & \multicolumn{4}{|c|}{ Typing of invasive cancers, n (\%) } & \multicolumn{3}{|c|}{ Grading of invasive cancers, $\mathrm{n}(\%)$} & \multirow[t]{2}{*}{ Total } \\
\hline & ductal & lobular & inflammatory & medullary & 1 & 2 & 3 & \\
\hline 1 & $30(32.6)$ & $3(30.0)$ & $4(80.0)$ & $0(0.0)$ & $21(36.2)$ & $4(44.4)$ & $12(29.3)$ & $37(34.3)$ \\
\hline 2 & $7(7.6)$ & $0(0.0)$ & $0(0.0)$ & $0(0.0)$ & $4(6.9)$ & $1(11.1)$ & $2(4.9)$ & $7(6.5)$ \\
\hline 3 & $47(51.1)$ & $5(50.0)$ & $1(20.0)$ & $0(0.0)$ & $28(48.3)$ & $4(44.4)$ & $21(51.2)$ & $53(49.1)$ \\
\hline 4 & $8(8.7)$ & $2(20.0)$ & $0(0.0)$ & $1(100)$ & $5(8.6)$ & $0(0.0)$ & $6(14.6)$ & $11(10.2)$ \\
\hline Total & $92(100)$ & $10(100)$ & $5(100)$ & $1(100)$ & $58(100)$ & $9(100)$ & $41(100)$ & $108(100)$ \\
\hline
\end{tabular}



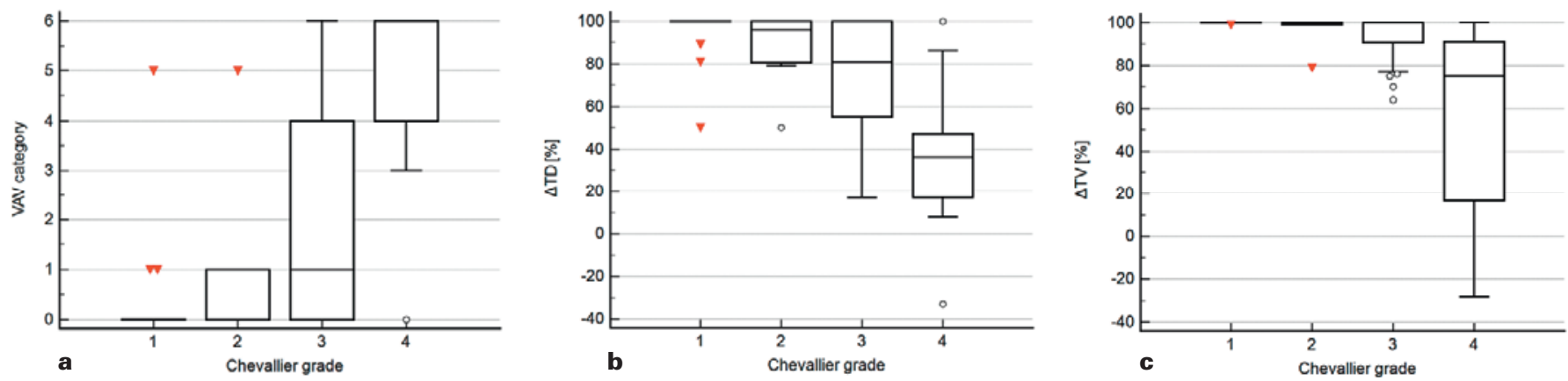

Fig. 2. Association of breast magnetic resonance imaging (bMRI) biomarkers with Chevallier grade. Given are box plots to explore the association of bMRI biomarkers with Chevallier grade ( $\triangle \mathrm{TD}$ : tumor diameter, $\triangle \mathrm{TV}$ : tumor volume). Volumetric analysis of vascularization (VAV) was graded from $\mathrm{VAV}$ to $\mathrm{VAV}$.

Table 3. Predictive accuracy of 3 different breast magnetic resonance imaging (bMRI) biomarkers for the assessment of tissue response to neoadjuvant chemotherapy (NAC)

\begin{tabular}{|c|c|c|c|c|c|c|}
\hline \multirow[t]{2}{*}{ Tissue response } & \multirow{2}{*}{$\begin{array}{l}\text { bMRI } \\
\text { biomarker }\end{array}$} & \multicolumn{5}{|c|}{ Predictive accuracy ${ }^{\mathrm{a}}$} \\
\hline & & Sensitivity & Specificity & $\mathrm{L}+$ & L- & AUC \\
\hline \multirow[t]{3}{*}{ Complete $^{\mathrm{b}}$} & VAV & \multirow{2}{*}{91.9} & \multirow{2}{*}{64.8} & \multirow{2}{*}{2.6} & \multirow{2}{*}{0.1} & 78.3 \\
\hline & $\Delta \mathrm{TD}$ & & & & & 79.5 \\
\hline & $\Delta \mathrm{TV}$ & 97.3 & 50.7 & 2.0 & 0.1 & 74.6 \\
\hline \multirow[t]{3}{*}{ Partial } & VAV & 53.3 & 83.7 & 3.3 & 0.6 & 68.6 \\
\hline & $\Delta \mathrm{TD}$ & 60.0 & 72.9 & 2.2 & 0.6 & 63.3 \\
\hline & $\Delta \mathrm{TV}$ & 43.3 & 77.1 & 1.9 & 0.7 & 58.1 \\
\hline \multirow[t]{3}{*}{ Non-response ${ }^{b}$} & VAV & \multirow{3}{*}{81.8} & 91.8 & 9.9 & 0.2 & 87.3 \\
\hline & $\Delta \mathrm{TD}$ & & 85.6 & 5.7 & 0.2 & 86.9 \\
\hline & $\Delta \mathrm{TV}$ & & 79.4 & 4.0 & 0.2 & 88.8 \\
\hline
\end{tabular}

${ }^{\mathrm{a}}$ Given are estimates of predictive accuracy.

${ }^{b}$ Regarding complete response and non-response to NAC, AUC levels of computer-aided diagnosis parameters were equal ( $\mathrm{p}=$ not significant).

AUC = Area under the curve; $\mathrm{L}+/ \mathrm{L}-=$ positive/negative likelihood ratio for bMRI biomarkers; $\Delta \mathrm{TD}=$ tumor diameter; $\triangle \mathrm{TV}=$ tumor volume; $\mathrm{VAV}=$ volumetric analysis of vascularization vs. tissue response to NAC (complete, partial, and non-response).
Partial responders (CG2 and CG3) showed different VAV patterns compared to complete and non-responders $(\mathrm{p}<0.001)$. Owing to substantial overlap of VAV patterns, these features were not able to differentiate CG2 from CG3 lesions ( $\mathrm{p}=\mathrm{n} . \mathrm{s}$.).

Most CG2 lesions were either $\mathrm{VAV}_{0}$ or $\mathrm{VAV}_{1}$ (each $42.9 \%, \mathrm{n}=$ $3)$. The 1 remaining case within this subgroup was $\operatorname{VAV}_{5}(14.3 \%)$. In the CG3 subgroup, $\operatorname{VAV}_{0}(39.6 \%, \mathrm{n}=21)$ and $\operatorname{VAV}_{3}(20.8 \%, \mathrm{n}=$ 11) were the most frequent vascularization patterns. The remaining CG3 cases ranged from $\operatorname{VAV}_{1}(13.2 \%, \mathrm{n}=7), \operatorname{VAV}_{4}(7.5 \%, \mathrm{n}=$ $4)$, and $\operatorname{VAV}_{5}(11.3 \%, \mathrm{n}=6)$ to $\operatorname{VAV}_{6}(7.5 \%, \mathrm{n}=4)$.

For VAV there was a positive correlation with $\mathrm{CG}(\mathrm{CG}=0.60$; $\mathrm{p}<0.001)$. Logistic regression identified typical VAV patterns for CG1 $\left(\mathrm{VAV}_{0}\right.$ : odds ratio $\left.(\mathrm{OR})=20.9\right)$ and $\mathrm{CG} 4\left(\mathrm{VAV}_{4}: \mathrm{OR}=33.4\right.$; $\mathrm{VAV}_{6}: \mathrm{OR}=66.8$; all $\left.\mathrm{p}<0.001\right)$. Figure 2 summarizes the association of $\Delta \mathrm{TD}, \Delta \mathrm{TV}$ VAV with CG.

\section{AUC Analysis}

Identification of patients with complete response showed similar values for all 3 parameters. Yet, $\Delta \mathrm{TD}$ reached the highest predictive accuracy (AUC $=79.5 \%$; table 3 ). Concerning partial response, VAV provided the highest AUC (68.6\%), L+ (3.3), and specificity (83.7\%). On the other hand, size measurement revealed lower accuracy for the assessment of lesions with partial response (AUC: $\Delta \mathrm{TD}=63.3 \%, \Delta \mathrm{TV}=58.1 \%$ ). Highest AUC values were observed for non-responders for all 3 parameters. Nevertheless, $\Delta \mathrm{TV}$ achieved the maximum AUC in the prediction of non-responders to NAC $(\mathrm{AUC}=88.8 \%)$.

\section{Discussion}

Semi-quantitative automated analysis of bMRI data enabled the prediction of tissue response to NAC. The automated softwarebased approach enabled us to investigate bMRI changes occurring due to cytotoxic treatment in a volumetric manner while avoiding observer-related bias. This is a clear advantage of our methodology over competing approaches to bMRI assessment of TR to NAC (e.g. [16] reporting $\kappa$ as low as 0.6 ).

Depending on the response category, we observed different levels of predictive accuracy. Generally, parameters showed similar levels of AUC (table 3). Nevertheless, in every category, 1 parameter showed optimal performance to differentiate TR. 
$\triangle \mathrm{TV}$ showed the highest accuracy in the assessment of non-response to NAC (AUC $=88.8 \%$ ). Accordingly, the strength of this parameter was the identification of small (volumetric) changes in tumor size, enabling the identification and differentiation of lesions with a TR of CG4 to NAC. On the other hand, $\triangle$ TD performed best in the assessment of complete response to NAC (AUC $=79.5 \%)$.

According to the study design, we can only speculate why $\Delta \mathrm{TD}$ slightly outperformed $\Delta \mathrm{TV}$ in the complete-response subgroup and $\Delta \mathrm{TV}$ surpassed $\Delta \mathrm{TD}$ in CG4 cases: In our experience, areas of non-mass enhancement are sometimes seen around vanishing tumors responding to NAC. The nature of these findings could be the upregulation of inflammatory pathways in order to promote the resorption of necrotic and apoptotic tumor tissue. Such findings are typically correlated with contrast uptake and can mimic real TV after NAC. This would lead to an overestimation of TV and result in decreased accuracy of $\Delta \mathrm{TV}$ in the assessment of CG1 to CG3 lesion.

Although both TV and TD measure the same target parameter (tumor size), the correlation between both values is not straightforward: The more the lesion leaves a classical spheric shape, and the more it changes to a non-mass like diffuse geometry, the less we would expect a straightforward correlation between $\Delta \mathrm{TV}$ and $\Delta \mathrm{TD}$. Since the abovementioned inflammatory enhancement around the vanishing tumor is typical non-mass like, this might serve as a potential hypothesis why $\Delta \mathrm{TD}$ outperformed $\Delta \mathrm{TV}$ in the case of residual tumor.

This argumentation might also explain why $\Delta \mathrm{TV}$ performed best within the CG4 subgroup. In these lesions, there was no response to treatment, and therefore this kind of enhancement is not likely to occur. It is all the more important to exactly match the size of the pre- to post-treatment bMRI, which is optimally performed in a volumetric way as provided by $\triangle \mathrm{TV}$.

The strength of VAV on the other hand was the differentiation of partial response from CG1 and CG4. This finding can be interpreted in the context of pathophysiology. Whereas complete and non-response to NAC is reflected by anatomical changes in the tumor through measurement of cancer size, assessment of partial response cannot be adequately done based on anatomical values but relies on functional parameters. Therefore, $\Delta \mathrm{TD}$ and $\Delta \mathrm{TV}$ were clearly inferior to VAV in this subgroup. Probably due to the presence of regressive tumor changes, $\triangle \mathrm{TD}$ again outperformed $\triangle \mathrm{TV}$. As VAV categorizes tumor vascularity, it is conclusive that VAV was most suitable to assess partial response to NAC.

Multiple studies have investigated the potential of bMRI to predict the degree of TR to NAC compared to mammography and ultrasound $[3,9,17,18]$. As this topic remained controversial, a recent meta-analysis summarized the current evidence [3]:

Compared to ultrasound, they identified a higher accuracy for bMRI in the prediction of tumor size after NAC. The researchers also identified substantial heterogeneity of ultrasound measurements compared to bMRI; this verifies the significant observer-related bias introduced by sonography in the assessment of TR. In this meta-analysis, the author reported that ultrasound could not be performed in 1 out of 10 patients of the study cohort, whereas bMRI was successfully performed in every patient. Based on such findings, one could expect a decreased applicability of breast ultrasound in the setting of TR assessment to NAC compared to bMRI. Nevertheless, this is not in accordance with our own experience, where ultrasound is certainly much easier to implement into the clinical workflow.

The authors also compared mammography with bMRI in the assessment of TR to NAC. In this cohort, all patients could be sufficiently assessed by bMRI. However, in 1 out of 4 patients (24.3\%), TR could not be evaluated by mammography. Typical reasons for problematic mammographies included dense breasts and dorsal tumor location.

Accordingly, the authors concluded that bMRI is superior to mammography and even ultrasound and should be considered the most appropriate test in the assessment of TR to NAC [3].

With this question having been answered, the most important task is now to determine how to optimally interpret and document bMRI findings in the NAC setting. The most common method in previous research was the manual measurement of lesions by the radiologist. This approach increases the likelihood of observer-related bias. The use of CAD can overcome this source of error as previously demonstrated $[17,18]$. Surprisingly, only few papers have applied this approach in assessing TR to NAC (for instance $[9,17,18])$.

Kim et al. [9] investigated whether TR can be predicted by CAD. They simplified the CG score and dichotomized patients into categories of 'complete response' and 'non-complete-response' to NAC. Different to our approach, 'non-complete-response' was not further categorized into CG2 to CG4. The authors reported a significant correlation of VAV with TR. Different from our analysis, they did not aim to quantify the accuracy of bMRI for the prediction of TR to NAC. This is why sensitivity, specificity, and AUC levels are not available.

Lyoung et al. [17] compared the performance of CAD with the assessment by an experienced radiologist. Their study collective was much smaller (57 lesions) compared to that of Kim et al. [9] (n $=148)$ and the present investigation $(\mathrm{n}=108)$. Similar to our data and different from Kim et al. [9], Lyoung et al. [17] documented the degree of TR on a much more detailed, 4-tailed, ordinal scale. Based on this analysis, the authors were able to verify that CAD is sufficiently accurate in the assessment of residual extent in breast cancer patients treated with NAC [17]. Yet, owing to the study rationale, no details were given on the accuracy of bMRI in the prediction of TR.

Although previous publications on the role of CAD in the assessment of TR had clearly different rationales, they agreed on the important role of VAV (for instance $[9,17]$ ). This is in accordance with our results: As shown in table 3, VAV was a powerful predictive parameter of TR. In answering the clinically most relevant questions (Complete response? Non-response?) performance was in the range of classical size measurements of tumor diameter and volume. In the more challenging prediction of 'partial response', VAV demonstrated even higher predictive values compared to 
$\Delta \mathrm{TD}$ and $\Delta \mathrm{TV}$. In our opinion, this indicates its potential to further improve the predictive accuracy of bMRI. This is also in accordance with previous research on prognostic bMRI, where VAV results were identified as tissue biomarkers and surrogates of patient outcome in breast cancer [6-8].

Although the levels of predictive accuracy in the current study are encouraging, they do not allow for histopathology or even surgical resection to be omitted. Therefore, the performance of bMRI in the assessment of TR must be improved. Diffusion weighted sequences are the most promising for this task. Previous work (for instance [19]) has demonstrated equal or even superior performance of diffusion weighted imaging compared to a standard dynamic contrast-enhanced protocol. As combinations of multiple imaging criteria [11] and multiparametric imaging have been shown to increase the accuracy of bMRI $[20,21]$, this approach should be further investigated in the assessment of TR to NAC in the near future.
In conclusion, we demonstrated the feasibility of a semi-quantitative automated analysis of bMRI, enabling the prediction of TR to NAC. The strength of this imaging biomarker was a standardized automatic interpretation of bMRI limiting observer-related bias. Hereby, parameters of tumor dimension and tissue vascularization were identified as imaging biomarkers for the assessment of TR to cytotoxic treatment. Future research should i) focus on the multiparametric assessment of bMRI in the NAC setting including DWI, and ii) translate such findings into multicentric oncological trials.

\section{Disclosure Statement}

No potential conflicts of interest were disclosed.

\section{References}

1 Mieog JSD, van der Hage JA, van de Velde CJH: Preoperative chemotherapy for women with operable breast cancer. Cochrane Database Syst Rev 2007; CD005002.

2 Kreienberg R, Albert S, Folkman M, et al.: Interdisziplinäre S3-Leitlinie für die Diagnostik, Therapie und Nachsorge des Mammakarzinoms. Langversion 3.0, Aktualisierung 2012 AWMF-Register-Nummer: 032-045OL 2012 (cited 01 August 2017); https://www. senologie.org/fileadmin/downloads/S3-Brustkrebsv2012-OL-Langversion.pdf.

3 Marinovich ML, Macaskill P, Irwig L, et al.: Meta-analysis of agreement between MRI and pathologic breast tumour size after neoadjuvant chemotherapy. $\mathrm{Br}$ Cancer 2013;109:1528-1536.

4 Baltzer PA, Dietzel M, Vag T, et al.: Can color-coded parametric maps improve dynamic enhancement pattern analysis in MR mammography? RöFo Fortschritte Auf Dem Geb Röntgenstrahlen Nukl 2010;182:254-260.

5 Baltzer PAT, Freiberg C, Beger S, et al.: Clinical MRmammography: are computer-assisted methods superior to visual or manual measurements for curve type analysis? A systematic approach. Acad Radiol 2009;16: 1070-1076.

6 Dietzel M, Zoubi R, Vag T, et al.: Association between survival in patients with primary invasive breast cancer and computer aided MRI. J Magn Reson Imaging 2013;37:146-155.

7 Baltzer PA, Zoubi R, Burmeister HP, et al.: Computer assisted analysis of MR-mammography reveals association between contrast enhancement and occurrence of distant metastasis. Technol Cancer Res Treat 2012; 11:553-560.
8 Baltzer PAT, Vag T, Dietzel M, et al.: Computer-aided interpretation of dynamic magnetic resonance imaging reflects histopathology of invasive breast cancer. Eur Radiol 2010;20:1563-1571.

9 Kim H, Kim HH, Park JS, et al.: Prediction of pathological complete response of breast cancer patients undergoing neoadjuvant chemotherapy: usefulness of breast MRI computer-aided detection. Br J Radiol 2014;87:20140142.

10 Morris EA, Comstock C, Lee C, et al.: ACR BI-RADS ${ }^{\circledR}$ magnetic resonance imaging; in ACR BI-RADS ${ }^{\circledR}$ Atlas, Breast Imaging Reporting and Data System, 5th ed. Reston, VA, American College of Radiology, 2013.

11 Baltzer PAT, Dietzel M, Kaiser WA: A simple and robust classification tree for differentiation between benign and malignant lesions in MR-mammography. Eur Radiol 2013;23:2051-2060.

12 Chevallier B, Roche H, Olivier JP, Chollet P, Hurteloup $\mathrm{P}$ : Inflammatory breast cancer. Pilot study of intensive induction chemotherapy (FEC-HD) results in a high histologic response rate. Am J Clin Oncol 1993;16: 223-228.

13 Szentmártoni G, Tőkés A-M, Tőkés T, et al.: Morphological and pathological response in primary systemic therapy of patients with breast cancer and the prediction of disease free survival: a single center observational study. Croat Med J 2016;57:131-139.

14 Mann RM, Kuhl CK, Kinkel K, Boetes C: Breast MRI: guidelines from the European Society of Breast Imaging. Eur Radiol 2008;18:1307-1318.
15 Baltzer PAT, Zoubi R, Burmeister HP, et al.: Computer assisted analysis of MR-mammography reveals association between contrast enhancement and occurrence of distant metastasis. Technol Cancer Res Treat 2012;11: 553-560.

16 Preibsch H, Wanner L, Bahrs SD, et al.: Background parenchymal enhancement in breast MRI before and after neoadjuvant chemotherapy: correlation with tumour response. Eur Radiol 2016;26:1590-1596.

17 Lyou CY, Cho N, Kim SM, et al.: Computer-aided evaluation of breast MRI for the residual tumor extent and response monitoring in breast cancer patients receiving neoadjuvant chemotherapy. Korean J Radiol 2011;12:34-43.

18 Takeda K, Kanao S, Okada T, et al.: Assessment of CAD-generated tumor volumes measured using MRI in breast cancers before and after neoadjuvant chemotherapy. Eur J Radiol 2012;81:2627-2631.

19 Woodhams R, Kakita S, Hata H, et al.: Identification of residual breast carcinoma following neoadjuvant chemotherapy: diffusion-weighted imaging - comparison with contrast-enhanced MR imaging and pathologic findings. Radiology 2010;254:357-366.

20 Pinker K, Bogner W, Baltzer P, et al.: Improved differentiation of benign and malignant breast tumors with multiparametric 18fluorodeoxyglucose positron emission tomography magnetic resonance imaging: a feasibility study. Clin Cancer Res Off J Am Assoc Cancer Res 2014;20:3540-3549.

21 Baltzer A, Dietzel M, Kaiser CG, Baltzer PA: Combined reading of contrast enhanced and diffusion weighted magnetic resonance imaging by using a simple sum score. Eur Radiol 2016;26:884-891. 